\title{
On Exact Solutions of Second Order Nonlinear Ordinary Differential Equations
}

\author{
Amjed Zraiqat ${ }^{1}$, Laith K. Al-Hwawcha ${ }^{2}$ \\ ${ }^{1}$ Al-Zaytoonah University of Jordan, Amman, Jordan \\ ${ }^{2}$ German Jordanian University, Amman, Jordan \\ Email: amjad@zuj.edu.jo, Laith.hawawsheh@qju.edu.jo
}

Received 26 April 2015; accepted 30 May 2015; published 2 June 2015

Copyright (C) 2015 by authors and Scientific Research Publishing Inc.

This work is licensed under the Creative Commons Attribution International License (CC BY).

http://creativecommons.org/licenses/by/4.0/

c) (i) Open Access

\begin{abstract}
In this paper, a new approach for solving the second order nonlinear ordinary differential equation $y^{\prime \prime}+p(x ; y) y^{\prime}=G(x ; y)$ is considered. The results obtained by this approach are illustrated by examples and show that this method is powerful for this type of equations.
\end{abstract}

\section{Keywords}

\section{Nonlinear Ordinary Differential Equation, Partial Differential Equation, Riccati Differential Equation}

\section{Introduction}

Exact solutions have always played and still play an important role in properly understanding the qualitative features of many phenomena and processes in various fields of natural science. Exact solutions of nonlinear equations, including those without a clear physical sense which do not correspond to real phenomena and processes, play an important role of test problems for verifying the correctness and assessment of accuracy of various numerical, asymptotic, and approximate methods. Moreover, the model equations admitting exact solutions serve as the basis for the development of new numerical, asymptotic, and approximate methods, which, in turn, enable us to study more complicated problems having no analytical solutions [1]. In the paper [2], Laith and Nama introduced a new approach for solving second order linear differential equation with variable coefficients

$$
y^{\prime \prime}+p(x) y^{\prime}+q(x) y=g(x) .
$$

To look for exact solution of (1) the authors introduced the substitution

$$
v(x)=y^{\prime}+\beta(x) y
$$


and have looked for a solution of the Riccati equation

$$
\beta^{\prime}=q(x)-p(x) \beta+\beta^{2} .
$$

In this paper, we generalize the idea of [2] and propose a general approach for solving the nonlinear second order equation

$$
y^{\prime \prime}+p(x, y) y^{\prime}+q(x, y) y=g(x, y)
$$

which can be written as

$$
y^{\prime \prime}+p(x, y) y^{\prime}=G(x, y)
$$

where $G(x, y)-g(x, y)-q(x, y) y$.

\section{The Main Results}

In this section, we propose an algorithm that enables us to reduce the Equations (4) and (5) by looking for solutions of the partial differential equations

$$
\begin{aligned}
& v_{x}(x, y)+(v(x, y)-\beta(x, y) y) v_{y}(x, y)=g(x, y)+\left(\beta_{y}(x, y) y-p(x, y)+\beta(x, y)\right) v(x, y) \\
& \beta_{x}(x, y)-y \beta(x, y) \beta_{y}(x, y)=q(x, y)-p(x, y) \beta(x, y)+\beta^{2}(x, y) .
\end{aligned}
$$

Theorem 1. If $v(x ; y)$ is any solution of (6) where $(x ; y)$ is a solution of (7), then Equation (4) can be reduced to a first order equation.

Proof. In order to prove this theorem, consider the transformation

$$
v(x)=y^{\prime}+\beta(x) y
$$

if we differentiate both sides of (8) with respect to $x$ we obtain

$$
v_{x}(x, y)+y^{\prime} v_{y}(x, y)=y^{\prime \prime}+\beta(x, y) y^{\prime}+\beta_{x}(x, y) y+\beta_{y}(x, y) y^{\prime} y
$$

substituting (4) and (8) in (9), we have

$$
\begin{aligned}
& v_{x}(x, y)+(v(x, y)-\beta(x, y) y) v_{y}(x, y) \\
& =g(x, y)+\left(\beta_{x}(x, y)-q(x, y)\right) y+\left(\beta_{y}(x, y) y-p(x, y)+\beta(x, y)\right) y^{\prime}
\end{aligned}
$$

assuming that $\beta(x, y)$ is a solution of (7), Equation (10) can be reduced to (6), solving (6) for $v(x, y)$ we have the result.

Theorem 2. If $v(x, y)$ is any solution of the equation

$$
v_{x}(x, y)+v(x, y) v_{y}(x, y)=G(x, y)-p(x, y) v(x, y) .
$$

Then (5) can be reduced to a first equation.

Proof. From theorem (1) the associated equation with $\beta(x, y)$ is

$$
\beta_{x}(x, y)-y \beta(x, y) \beta_{y}(x, y)=p(x, y) \beta(x, y)+\beta^{2}(x, y)
$$

which has a solution $\beta(x, y)=0$, thus the equation associated with $v(x, y)$ is (11), solving for $v(x, y)$ Equation (5) reduced to a first order equation.

Theorem 3. If $\beta(x, y)$ is any solution of the equation

$$
\beta_{x}(x, y)-y \beta(x, y) \beta_{y}(x, y)=\frac{-G(x, y)}{y}-p(x, y) \beta(x, y)+\beta^{2}(x, y), y \neq 0 .
$$

Then Equation (5) can be reduced to first order equation.

Proof. Equation (5) can be written as

$$
y^{\prime \prime}+p(x, y) y^{\prime}-\frac{G(x, y)}{y} y=0
$$


applying theorem (1), we have that $v(x, y)=0$ is a solution of

$$
v_{x}(x, y)+(v(x, y)-\beta(x, y) y) v_{y}(x, y)=(\beta(x, y) y-p(x, y)+\beta(x, y)) v(x, y)
$$

solving (13) for $\beta(x, y)$, the result follows.

Theorem 4. If $\frac{\partial p(x, y)}{\partial x}=-\frac{\partial G(x, y)}{\partial y}$, then Equation (5) can be reduced to a first order equation.

Proof. Applying theorem (2) the result follows.

\section{Examples}

In this section, we give some examples on our approach for reduction and finding solutions of nonlinear second order ordinary differential equations, these equations and more equations that can be easily solved by this method can be found in [1] [3]-[7].

Example 1. Consider the equation

$$
y^{\prime \prime}=f(y)
$$

comparing with Equation (4) we note that $p(x, y)=0, q(x, y)=0, g(x, y)=f(y)$.

First, we solve

$$
\beta_{x}(x, y)-y \beta(x, y) \beta_{y}(x, y)=\beta^{2}(x, y)
$$

the associated ratios with Equation (17) are

$$
\frac{\mathrm{d} x}{1}=\frac{\mathrm{d} y}{-\beta_{y}}=\frac{\mathrm{d} \beta}{\beta^{2}}
$$

from which, we find that $\beta(x, y)=\frac{c_{1}}{y}$.

Second, we solve

$$
v_{x}(x, y)+\left(v(x, y)-c_{1}\right) v_{y}(x, y)=f(y)
$$

the associated ratios with Equation (19) are

$$
\frac{\mathrm{d} x}{1}=\frac{\mathrm{d} y}{v-c_{1}}=\frac{\mathrm{d} v}{f(y)}
$$

from which, we find that $(x y)=c_{1} \pm\left(c_{2}+2 \int f(y) \mathrm{d} y\right)^{\frac{1}{2}}$.

Finally, we substitute $\beta(x, y), v(x, y)$ in Equation (8) to get

$$
x \pm c_{3}=\int\left(c_{2}+2 \int f(y) \mathrm{d} y\right)^{\frac{1}{2}} .
$$

Example 2. Consider the equation

$$
x y^{\prime \prime}=n y^{\prime}+x^{2 n+1} f(y)
$$

this equation can be written as

$$
y^{\prime \prime}-\frac{n}{x} y^{\prime}=x^{2 n} f(y)
$$

comparing with Equation (5) we have that $p(x, y)=\frac{-n}{x}, q(x, y)=0, G(x, y)=x^{2 n} f(y)$.

The equation associated with $\beta(x, y)$ is

$$
\beta_{x}(x, y)-y \beta_{y}(x, y)=\frac{n}{x} \beta+\beta^{2}
$$


from which we find that $\beta(x, y)=0$. The equation associated with $v(x, y)$ is

$$
v_{x}(x, y)+v(x, y) v_{y}(x, y)=x^{2 n} f(y)+\frac{n}{x} v
$$

we look for a solution of the form

$$
v(x, y)=m(x) n(y)
$$

substituting $v(x, y)$ in Equation (25), we have

$$
m^{\prime}(x) n(y)+m^{2}(x) n(y) n^{\prime}(y)=x^{2 n} f(y)+\frac{n}{x} m(x) n(y) .
$$

Thus, $m(x)$ and $n(y)$ must satisfy the following equations

from which we find that

$$
\begin{aligned}
& m^{\prime}(x)=\frac{n}{x} m(x) \\
& m^{2}(x)=x^{2 n} \\
& n(y) n^{\prime}(y)=f(y)
\end{aligned}
$$

$$
\begin{aligned}
& m(x)=x^{n} \\
& n(y)= \pm\left(c_{1}+2 \int f(y) \mathrm{d} y\right)
\end{aligned}
$$

so, $v(x, y)= \pm x^{n}\left(c_{1}+2 \int f(y) \mathrm{d} y\right)^{\frac{1}{2}}$. Finally we solve

$$
y^{\prime}= \pm x^{n}\left(c_{1}+2 \int f(y) \mathrm{d} y\right)^{\frac{1}{2}}
$$

and two cases are considered,

$$
\begin{aligned}
& n=-1 \text {, the solution is } \int\left(c_{1}+2 \int f(y) \mathrm{d} y\right)^{\frac{1}{2}}= \pm \ln |x|+c_{2} \\
& n \neq-1 \text {, the solution is } \int\left(c_{1}+2 \int f(y) \mathrm{d} y\right)^{\frac{1}{2}} \mathrm{~d} y= \pm \frac{x^{2 n+1}}{n+1}+c_{2} .
\end{aligned}
$$

Example 3. Consider the equation

Equation (36) can be written as

$$
(1-y)^{2} y^{\prime \prime}-\left(x+\frac{3}{2}\right) y^{\prime}-=1-y
$$

$$
y^{\prime \prime}-\frac{\left(x+\frac{3}{2}\right)}{(1-y)^{2}} y^{\prime}-=\frac{1}{1-y}
$$

Comparing with Equation (5) we have $p(x, y)=\frac{\left(x+\frac{3}{2}\right)}{(1-y)^{2}}, G(x, y)=\frac{1}{1-y}$, furthermore $\frac{\partial_{p}(x, y)}{\partial x}=-\frac{\partial G(x, y)}{\partial y}$. So, theorem (4) can be applied as follows:

$$
v_{x}(x, y)=\frac{1}{1-y}
$$


which implies that

$$
v(x, y)=\frac{x}{1-y}+\psi(y) .
$$

Differentiating both sides of (39), we have

$$
v_{y}(x, y)=\frac{x}{(1-y)^{2}}+\psi^{\prime}(y) .
$$

Assuming that $v_{y}(x, y)=-p(x, y)$, yields

$$
\frac{\left(x+\frac{3}{2}\right)}{(1-y)^{2}}=\frac{x}{(1-y)^{2}}+\psi^{\prime}(y)
$$

thus, Equation (36) reduced to the first order exact ordinary differential equation

$$
y^{\prime}=\frac{2 x+3}{2-2 y}
$$

which has the solution

$$
x^{2}+3 x+y^{2}-2 y=C
$$

\section{Conclusion}

In this article, a new method is considered for solving second order nonlinear ordinary differential equations. The small size of computation in comparison with the computational size required by other analytical methods [1], and the dependence on first order partial differential equations show that this method can be improved and introduces a significant improvement in solving this type of differential equations over existing methods. This method is proposed to be considered as an alternative approach being employed to a wide variety of equations.

\section{References}

[1] Polyanin, A.D. and Zaitsev, V.F. (2003) Handbook of Exact Solutions for Ordinary Differential Equations. 2nd Edition, Chapman \& Hall/CRC Press, Boca Raton.

[2] Al-Hwawcha, L.K. and Abid, N.A. (2008) A New Approach for Solving Second Order Ordinary Differential Equations. Journal of Mathematics and Statistics, 4, 58-59. http://dx.doi.org/10.3844/jmssp.2008.58.59

[3] Polyanin, A.D. and Manzhirov, A.V. (2006) Handbook of Mathematics for Engineers and Scientists. Chapman \& Hall/ CRC Press, Boca Raton. http://dx.doi.org/10.1201/9781420010510

[4] Zwillinger, D. (1997) Handbook of Differential Equations. 3rd Edition, Academic Press, Boston.

[5] Polyanin, A.D., Zaitsev, V.F. and Moussiaux, A. (2002) Handbook of First Order Partial Differential Equations. Taylor \& Francis, London.

[6] Kamke, E. (1977) Differentialgleichungen: Losungsmethoden und Losungen. I. Gewohnliche Differentialgleichungen. B. G. Teubner, Leipzig. http://dx.doi.org/10.1007/978-3-663-05925-7

[7] Boyce, W.E. and Di Prima, R.C. (2000) Elementary Differential Equations and Boundary Value Problems. John Wiley and Sons, Inc., Hoboken. 